\title{
Acute Moraxella catarrhalis Airway Infection of Chronically Smoke-Exposed Mice Increases Mechanisms of Emphysema Development: A Pilot Study
}

\author{
Katja Fischer ${ }^{1,2}$, Jan-Moritz Doehn ${ }^{1}$, Christian Herr ${ }^{3}$, Carolin Lachner ${ }^{2}$, Annina Heinrich ${ }^{2}$, \\ Olivia Kershaw ${ }^{4}$, Meike Voss ${ }^{3}$, Max H. Jacobson ${ }^{5}$, Achim D. Gruber ${ }^{4}$, Matthias Clauss ${ }^{6}$, \\ Martin Witzenrath $^{1,7}$, Robert Bals ${ }^{3}$, Birgitt Gutbier ${ }^{1,7}$ and Hortense Slevogt ${ }^{2 *}$ \\ 'Charité - Universitätsmedizin Berlin, corporate member of Freie Universität Berlin, Humboldt-Universität zu Berlin, \\ and Berlin Institute of Health, Department of Infectious Diseases and Respiratory Medicine, Berlin, Germany \\ ${ }^{2}$ Septomics Research Center, Jena University Hospital, Jena, Germany \\ ${ }^{3}$ Department of Internal Medicine V-Pulmonology, Allergology, Respiratory Intensive Care Medicine, \\ University of the Saarland, Homburg Saar, Germany \\ Department of Veterinary Pathology, Freie Universität, Berlin, Germany \\ ${ }^{5}$ Pathology and Laboratory Medicine, IU School of Medicine, Indianapolis, Indiana, USA \\ ${ }^{6}$ Indiana Center for Vascular Biology and Medicine and Department of Cellular and Integrative Physiology, \\ Indiana University, Indianapolis, Indiana, USA \\ 'Charité - Universitätsmedizin Berlin, corporate member of Freie Universität Berlin, Humboldt-Universität zu Berlin, \\ and Berlin Institute of Health, Division of Pulmonary Inflammation, Berlin, Germany
}

Received: 30 July 2018; accepted: 07 September 2018

\begin{abstract}
In chronic obstructive pulmonary disease (COPD), acute exacerbations and emphysema development are characteristics for disease pathology. COPD is complicated by infectious exacerbations with acute worsening of respiratory symptoms with Moraxella catarrhalis as one of the most frequent pathogens. Although cigarette smoke (CS) is the primary risk factor, additional molecular mechanisms for emphysema development induced by bacterial infections are incompletely understood. We investigated the impact of $M$. catarrhalis on emphysema development in CS exposed mice and asked whether an additional infection would induce a solubilization of pro-apoptotic and proinflammatory endothelial monocyte-activating-protein-2 (EMAPII) to exert its activities in the pulmonary microvasculature and other parts of the lungs not exposed directly to CS.

Mice were exposed to smoke (6 or 9 months) and/or infected with $M$. catarrhalis. Lungs, bronchoalveolar lavage fluid (BALF), and plasma were analyzed.

CS exposure reduced ciliated area, caused rarefaction of the lungs, and induced apoptosis. EMAPII was increased independent of prior smoke exposure in BALF of infected mice. Importantly, acute $M$. catarrhalis infection increased release of matrixmetalloproteases-9 and -12, which are involved in emphysema development and comprise a mechanism of EMAPII release.

Our data suggest that acute $M$. catarrhalis infection represents an independent risk factor for emphysema development in smoke-exposed mice.
\end{abstract}

Keywords: chronic smoke exposure, COPD, M. catarrhalis, emphysema, EMAPII

\section{Introduction}

Chronic obstructive pulmonary disease (COPD) is characterized by chronic bronchitis associated with chronic inflammation of the small airways and in the lung parenchyma, as well as obstruction of the small airways [1]. Cigarette smoke (CS) has been shown to impair ciliary functions of human airway epithelial cells as well as in murine models of long-term smoke exposure [2, 3]. Destruction of the mucociliary clearance is a main cause of pathological bacterial colonization followed by low-grade airway inflammation and by an increased frequency of acute exacerbations (AECOPD) [4]. CS is also associated with the development of emphysema, the major consequence of COPD severity, which also correlates with the degree of progressive pulmonary inflammation $[5,6]$. The development of emphysema has been found to be accelerated

\footnotetext{
*Author for correspondence: Septomics Research Center, Jena University Hospital, Albert-Einstein Str. 10, 07745 Jena, Germany; Email: E-mail: hortense. slevogt@med.uni-jena.de; Tel: +49 36419396520.
}

due to the release of metalloproteases (MMPs) and other proteolytic enzymes into the lungs [7]. In addition to protease imbalance leading to loss of tissue structure, loss of tissue itself is another hallmark of emphysema [8]. Another mechanism in the emphysema development has been shown to be associated with caspase-dependent alveolar septal cell apoptosis [9]. Endothelial monocyte-activating protein 2 (EMAPII) also plays an important role in the development of smoke induced emphysema and transgenic lung specific expression of secreted EMAPII causes pulmonary apoptosis, alveolar rarefaction, and MMP9 and MMP12 upregulation [10].

Bacterial infections are known to induce increased levels of the extracellular metalloproteases such as MMP9 and MMP12 [11], which have been demonstrated to be elevated in mice exposed to CS $[12,13]$, with MMP9 amongst others responsible for inducing the secretion of EMAPII.

M. catarrhalis is isolated from the sputum in about $25 \%$ of acute exacerbations in COPD patients [14], while being a rare event in healthy individuals. Recently, we could demonstrate

This is an open-access article distributed under the terms of the Creative Commons Attribution-NonCommercial 4.0 International License (https://creativecommons.org/licenses/by-nc/4.0/), which permits unrestricted use, distribution, and reproduction in any medium for non-commercial purposes, provided the original author and source are credited, a link to the CC License is provided, and changes - if any - are indicated. 
that $M$. catarrhalis induced acute inflammatory immune responses in lungs of infected and long-term smoke-exposed mice [15]. Importantly, the frequency of AECOPD has lately been identified to be an important independent risk factor for emphysema development in COPD patients [16]. However, little is known about the mechanisms of emphysema development in response to acute bacterially induced exacerbations. This is surprising, since bacteria are known to be able to increase expression of MMPs [17] and induce apoptosis in in vitro cellular models [18].

In this study, we aimed to investigate if acute infections of $M$. catarrhalis in a murine model of smoke-induced COPD contribute to the induction of emphysema development.

\section{Materials and Methods}

Animals. C57BL/6N mice were maintained under specific pathogen-free conditions in accordance with the European Guidelines for Animal Welfare. Animals were housed with unlimited access to food and water. To reduce interindividual variation caused by using different sex and age, we performed the infection experiments without prior smoke exposure using female mice at the age of 11-14 weeks. For subsequent smoke exposure, mice at the age of 9 weeks were obtained. All experiments were approved by institutional and governmental authorities (Landesamt für Gesundheit und Soziales Berlin; Landesamt für Soziales, Gesundheit und Verbraucherschutz, Saarbrücken).

Exposure to Cigarette Smoke. Female C57BL/6N mice were whole-body exposed at the age of 9 weeks (wk) to mainstream and side-stream cigarette smoke using a smoking chamber (Model TE-10 Teague Enterprises, Davis, CA). Control groups were exposed to ambient air. Smoke-treated animals were exposed to cigarette smoke from research cigarettes (University of Kentucky, Reference Cigarette Program) at a concentration of $140 \mathrm{mg} / \mathrm{m}^{3}$ (TSP) $5 \mathrm{~h} /$ day, 5 days/wk for up to 9 months. An inspection of the concentration was performed on a weekly basis by the means of gravitational measurements.

Processing for Electron Microscopy. Lung tissue was prepared for electron microscopy as described in a previous study [19]. Samples were fixed in $2.5 \%$ glutaraldehyde in $0.06 \mathrm{M}$ phosphate buffer (PB, pH 7.3) for up to 2 days. Following dehydration in a graded series of alcohol concentrations, the tissue was immersed in hexamethyldisilazane (HMDS) (Sigma; $2 \times 10 \mathrm{~min}$ ), dried, and sputter-coated with gold (BAL-TEC MED 020), and scanning electron microscopy (SEM) was performed in a DSM 982 Gemini Zeiss microscope.

At least four scanning electron images of murine bronchial epithelium gained from 3-5 different individuals per group (magnification: 2000×) were analyzed regarding the area covered by cilia in relation to the total area. Measurements were performed by determination of pixel count per area.

Lung Histology. After excision, the lungs were prepared for further evaluation of histological lung morphology. Sections were stained with hematoxylin/eosin or caspase-3antibody (caspase-3 active, R\&D Systems), or a PAS-reaction was performed using a routine staining procedure. Descriptive analyses were performed including a scoring system, modified from the score used by Zeldin et al. (2001) [20].

Bacterial Strain and Culture. M. catarrhalis strain BBH18 was grown, harvested, and resuspended before inoculation as described in our previous study [15].

Bacterial Challenge. Animals were anesthetized by intraperitoneal injection of ketamine $(80 \mathrm{mg} / \mathrm{kg})$ and xylazine $(25 \mathrm{mg} / \mathrm{kg}$ ). Transoral intratracheal intubation was performed with a 24-gauge intravenous catheter, and bacterial suspension was instilled intratracheally as described in our previous study [15]. The clinical conditions, rectal temperature, and body weight were monitored twice per day.

Protein Quantification in Bronchoalveolar Lavage Fluid (BALF) and Plasma. BAL was performed twice with $800 \mu \mathrm{L}$ of ice-cold phosphate-buffered saline (PBS) containing protease inhibitor (complete Mini Roche). Only the first, more concentrated wash was processed for enzyme-linked immunosorbent assay (ELISA), and the supernatant was analyzed after spinning. Blood was withdrawn from the vena cava and centrifuged for $10 \mathrm{~min}$. Plasma and the supernatant of the BALF analyzed by ELISA according to the manufacturer's instructions (EMAPII: MyBioSource (\#MBS268308), San Diego, USA; MMP9: Boster Biological Technology Co., Ltd (\#EK0466); MMP12: Antikoerper-online.de (\#ABIN415783) and cytokine multiplex assay (Bioplex ${ }^{\circledR}$, Bio-Rad Laboratories).

EMAPII Staining for Average Positivity. EMAPII histology was performed as previously described for a recent study from Clauss et al. with modifications [10]. Briefly, paraffin-sections from mouse lungs were deparaffinized by heating at $60{ }^{\circ} \mathrm{C}$ for $1 \mathrm{~h}$ followed by rehydration in descending ethanol line. Slides were cooked in citrate buffer $(0.01 \mathrm{M}, \mathrm{pH} 6)$ for $20 \mathrm{~min}$ and then permeabilized with $1 \%$ Triton (in $0.01 \mathrm{M}$ PBS) and blocked in a blocking buffer (PBS $0.01 \mathrm{~m} ; 1 \%$ BSA; $0.05 \%$ Tween 20). For EMAPII labeling, anti-EMAPII polyclonal rabbit antibody (SA86) at 1:500 dilution was applied overnight. After a wash step for $2 \mathrm{~h}$ in PBS, the slides were incubated with secondary antibody (goat anti-rabbit HRP at 1:2000 dilution) for 30 min. The slides were developed with NovaRED substrate kit and counterstained for $30 \mathrm{~s}$ with the hematoxylinQS/vector after washing. Finally, dehydration of slides was performed in ethanol (95\%), isopropanol, and xylene before embedding in mounting medium. The Aperio Positive Pixel Count Algorithm was used to quantify the amount of a specific stain present in a scanned slide image. A range of color (range of hues and saturation) and 3 intensity ranges (weak, positive, and strong) were masked and evaluated. The algorithm counted the number and intensity sum in each intensity range, along with three additional quantities: average intensity, ratio of strong/total number, and average intensity of weak positive pixels.

Cell Lines. Primary mouse alveolar epithelial cells were cultivated as recommended by the manufacturer. Cells were stimulated with Tumor necrosis factor alpha (TNF- $\alpha, 10 \mathrm{ng}$ ), CS-extract (CSE) (4\%), M. catarrhalis multiplicity of infection (MOI) 1 and MOI 10, as described for a recent study from Klaile et al. [21].

CSE was prepared as described for a recent study from Klaile et al. (2013): smoke from one cigarette (10 mg tar, $0.8 \mathrm{mg}$ nicotine; Camel Filters, Japan Tobaco International) with the mouthpiece filter removed was bubbled through 10-mL complete medium using a peristaltic pump (P-1, GE Healthcare) and filtered through a $0.22-\mu \mathrm{m}$ filter. Suction was regulated, so that side-stream smoke would develop during the entire combustion, lasting $5 \mathrm{~min}$ for each cigarette. The filtered CSE was regarded as $100 \%$. CSE was used within $30 \mathrm{~min}$ of preparation at a final concentration of $4 \%$ by adding $80-\mu \mathrm{L}$ CSE $(100 \%)$ to $1920-\mu \mathrm{L}$ medium per well containing 1-million cells [21].

Flow Cytometry. To analyze the total expression of EMAPII in primary mouse alveolar epithelial cells, the cells were fixed in Cytofix/Cytoperm solution (BD Biosciences) and treated with the rat anti-EMAPII monoclonal antibody M7/1 for $45 \mathrm{~min}$, followed by washing twice with Perm/ Wash. Then, the cells were incubated with the Millipore rat 
anti-EMAPII, rat Immunoglobulin $\mathrm{G}$ (IgG) isotype control antibody and corresponding secondary $\mathrm{F}(\mathrm{ab}) 2$ anti-rat phycoerythrin (PE) antibodies (1:50) for $30 \mathrm{~min}$ and washed twice with Perm/Wash and once with PBS. The samples were measured in FACSCalibur, and the data were analyzed using the CellQuest Pro software (BD Biosciences).

Statistical Analysis. Statistical analyses were performed using Graphpad PRISM 5.0 software and data were expressed as mean + standard error of mean (SEM). Statistical significance between different groups was analyzed using the Mann-Whitney $U$ and Kruskal-Wallis tests, as well as Dunn's test for post hoc comparisons. A $p$-value of 0.05 was considered statistically significant. Three independent in vivo experiments were performed, but every experiment was performed for different analysis. Separate experiments were performed for the histology and SEM analysis $(n=3-5)$, for the quantification of EMAPII and MMP-9/-12 concentrations in BALF and plasma $(n=3-6)$, and for the expression analysis of EMAPII and MMP-9/-12 in lung tissue $(n=3-5)$. The in vitro experiment with primary mouse alveolar epithelial cells was repeated three times, and a representative image was shown.

\section{Results}

Long-Term Smoke Exposure Leads to Reduction of Cilia Covering Bronchial Epithelium. To address the role of smoke-induced damage in the airways leading to reduced bacterial clearance, we analyzed the effect of long-term smoke exposure after 9 months on the area covered by ciliated bronchial epithelium. For this, we developed a new method based on electron microscopy to estimate the area covered by ciliated cells in relation to the total area (Figure 1A). Lung tissue was prepared for electron microscopy, comparable sections of bronchial epithelium were photographed, and the ciliated area in relation to total area was evaluated by determination of pixel count per area.

We observed a significant reduction to approximately $45 \%$ of bronchial epithelium covered by cilia in smoke-exposed mice compared to air control mice (Figure 1B). These findings suggest that cigarette smoke exposure could reduce the defense mechanism against infectious agents.
A

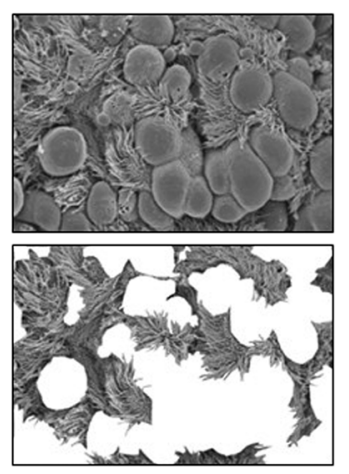

B

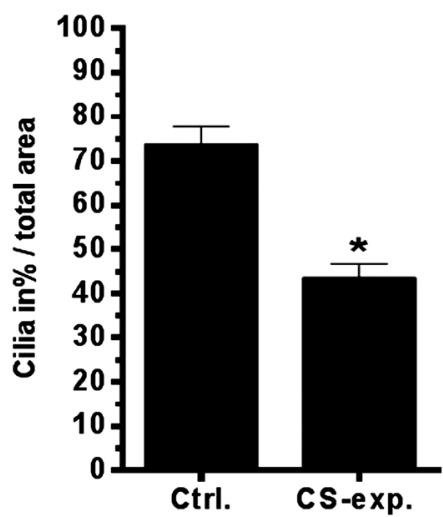

Figure 1. Long-term smoke exposure leads to reduction of cilia covering bronchial epithelium. (A) Example of scanning electron images of murine bronchial epithelium $(2000 \times)$ analyzed regarding the area covered by ciliated cells in relation to the total area. (B) Smoketreated animals were compared with control animals, using the Mann-Whitney $U$-test $\left({ }^{*} p<0.05\right)$. Bars represent mean + SEM of at least 4 pictures of visual fields, gained from 3-5 different individuals analyzed
Long-Term Smoke Exposure Leads to Development of Lung Damage and Emphysema and Induces Caspase-3 Expression in the Murine Lungs. Moreover, we investigated the effect of long-term smoke exposure on histopathological changes in the murine lungs. For the evaluation, we used a specific scoring system, developed by Zeldin et al. [20]. These evaluations include the estimation of the intensity of specific features like edema, inflammatory cell infiltration, and elevated numbers of alveolar macrophages, as well as goblet cell metaplasia and emphysema development in smokeexposed animals, in comparison to animals exposed to ambient air. Our data showed significantly increased specific lung damage in mice, long-term smoke-exposed over 6 and 9 months. Moreover, we observed increased emphysema development in the group of mice smoke-exposed for 9 months (Figure 2A-C and Figure S1).

To validate the involvement of apoptosis in our model, we employed caspase-3 as an apoptosis marker associated with lung emphysema [22]. Indeed, caspase- 3 expression was elevated in the lungs from three of the four animals that were smoke-exposed for 9 months, when compared to controls (Figure 2D and Figure S2). These findings recapitulate the basic features of human emphysema and enable us to study the role of exacerbating infectious agents on top of cigarette smoke exposure-induced emphysematous changes.

M. catarrhalis Increases EMAPII in BALF and Lung Tissue of Long-Term Smoke-Exposed Mice and in Primary Mouse Alveolar Epithelial Cells. Previously, Clauss et al have shown that a neutralizing antibody to EMAPII could ablate caspase-3 activation and emphysema progression in a murine model with cigarette smoke exposure $[8,10]$. Although they had found increased epithelial EMAPII expression in response to cigarette smoke exposure, it could not be explained how this localized expression could lead to vascular endothelial apoptosis previously identified to cause emphysema in mice [8]. Based on these data and our finding that the airway defense mechanism was decreased in cigarettesmoke-exposed mice, we tested the effect of $M$. catarrhalis infection on EMAPII expression and release in cigarettesmoke-exposed mice. Analyzing the release of EMAPII into the BALF by ELISA, we found that $M$. catarrhalis infection strongly and significantly increased EMAPII in the BALF of both cigarette-smoke-exposed and non-exposed mice (Figure 3A). Although cigarette smoke exposure alone did not result in significantly elevated levels of released EMAPII in the BALF, cell bound EMAPII protein expression in the murine lung tissue was significantly elevated when mice were exposed to cigarette smoke alone, as determined by immunohistochemistry (Figure 3B). Importantly, infection with $M$. catarrhalis decreased the cell bound positivity to a level comparable to the controls, suggesting that smoke exposure induced EMAPII is secreted into the airways in response to $M$. catarrhalis infection.

To replicate these finding in a cellular model, we could confirm our findings in in vitro experiments, in which we treated murine alveolar epithelial cells with $M$. catarrhalis. Indeed, we observed EMAPII secretion in response to M. catarrhalis (MOI 1 or 10) (Figure 3C). Interestingly, EMAPII was also released in response to CSE, suggesting that in vitro and in vivo models may address different mechanisms.

Acute M. catarrhalis Airway Infection Increases MMP9 and MMP12 Expression in the BALF and Plasma of Long-Term Smoke-Exposed Mice. MMP activation was described to be secreted in response to bacterial infections [10]. In addition, MMP activation was reported to cause release of cell bound EMAPII [23]. Therefore, we tested the hypothesis that acute $M$ catarrhalis infection increased MMP production to alter 

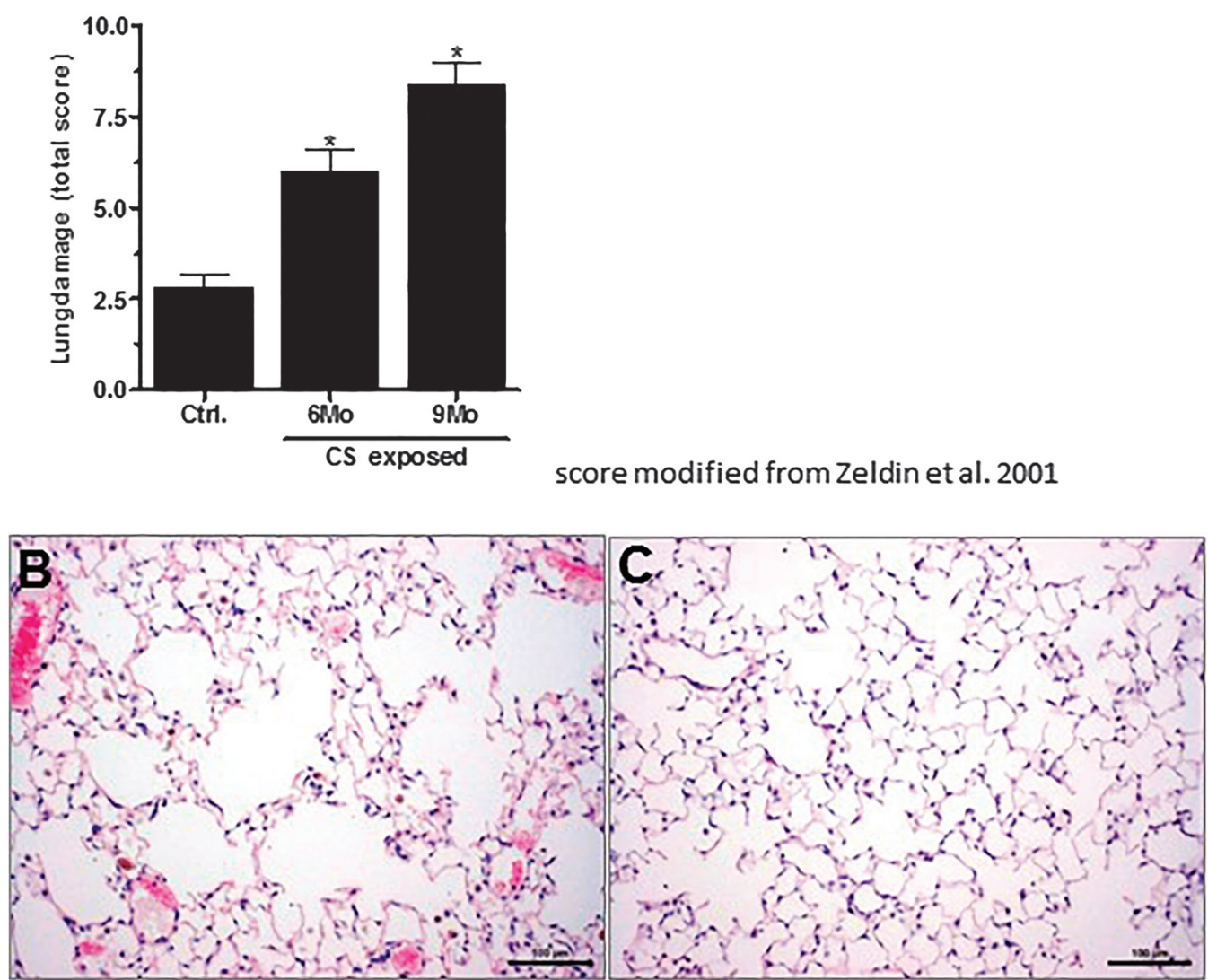

D

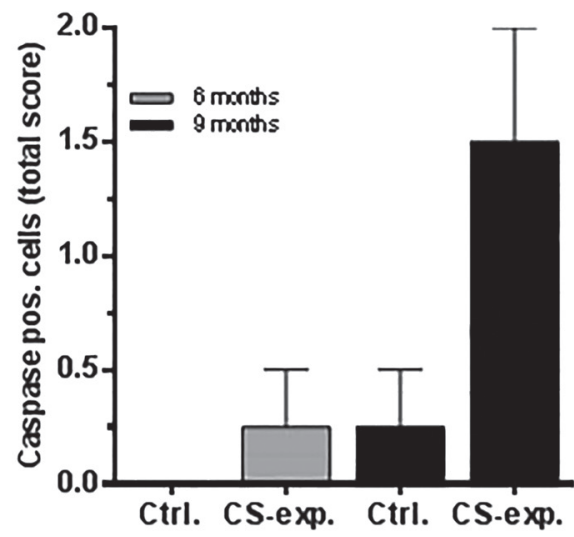

Figure 2. Long-term smoke exposure leads to development of lung damage and emphysema and induces caspase-3 expression in the murine lungs. (A) Total score of lung damage in control mice and mice exposed to cigarette smoke for 6 and 9 months, consistent of evaluation of accumulation of intracytoplasmic, brown pigments, and number of alveolar-macrophages in 10 High Power Fields (HPF), extent of alveolar lung emphysema, occurrence of perivascular edema, interstitial inflammatory cell infiltration, and perivascular, peribronchial, and subpleural inflammatory cell infiltration and goblet cell metaplasia $(n=5,3,5$, respectively). Smoke-exposed and control animals were compared using the Mann-Whitney $U$-test, and bars represent mean + SEM $(* p<0.05)$. Extent of alveolar lung emphysema in mice exposed to cigarette smoke for 9 months $(B)$ and controls (C). Pictures are representative for 3-5 analyzed lungs - magnification $100 \times$, bar $=100 \mu \mathrm{m}$. (D) Score of caspase-3 positive cells in the analyzed lung tissue of mice exposed to cigarette smoke for 6 and 9 months and control $(n=4)$, and bars represent mean + SEM. Control (Ctrl.) group, 6 months: no caspase expression was detectable in all four lungs (scored with 0); smoke-exposed (CSE) group, 6 month: no caspase expression was detectable in 3 of 4 lungs and, in one lung, 1 localization of caspase positive cells (scored with 1) was found; Ctrl. group, 9 month: no caspase expression was detectable in 3 of 4 lungs and, in one lung, 1 localization of caspase positive cells was found; CS-exp. group 9 month: in 3 of 4 lungs multifocal accumulated caspase-positive cells (scored with 2) were detectable and no caspase expression was found in one lung

the progression of emphysema. We analyzed expression of MMP9 and 12 in the BALF and plasma of animals after infection with $M$. catarrhalis with or without prior smoke exposure or with smoke exposure alone compared to control animals (Figure 4AD). Smoke exposure alone did not contribute to increased levels of MMP9 and 12 in the BALF. In contrast, we found that
MMP12 expression was elevated in response to M. catarrhalis infection, regardless of prior smoke exposition (Figure 4B). In addition, significantly elevated MMP9 and 12 expressions were found in the plasma of solely smoke-exposed animals after 9 months and animals that received an additional M. catarrhalis infection after long-term smoke exposure (Figure 4C, D). 

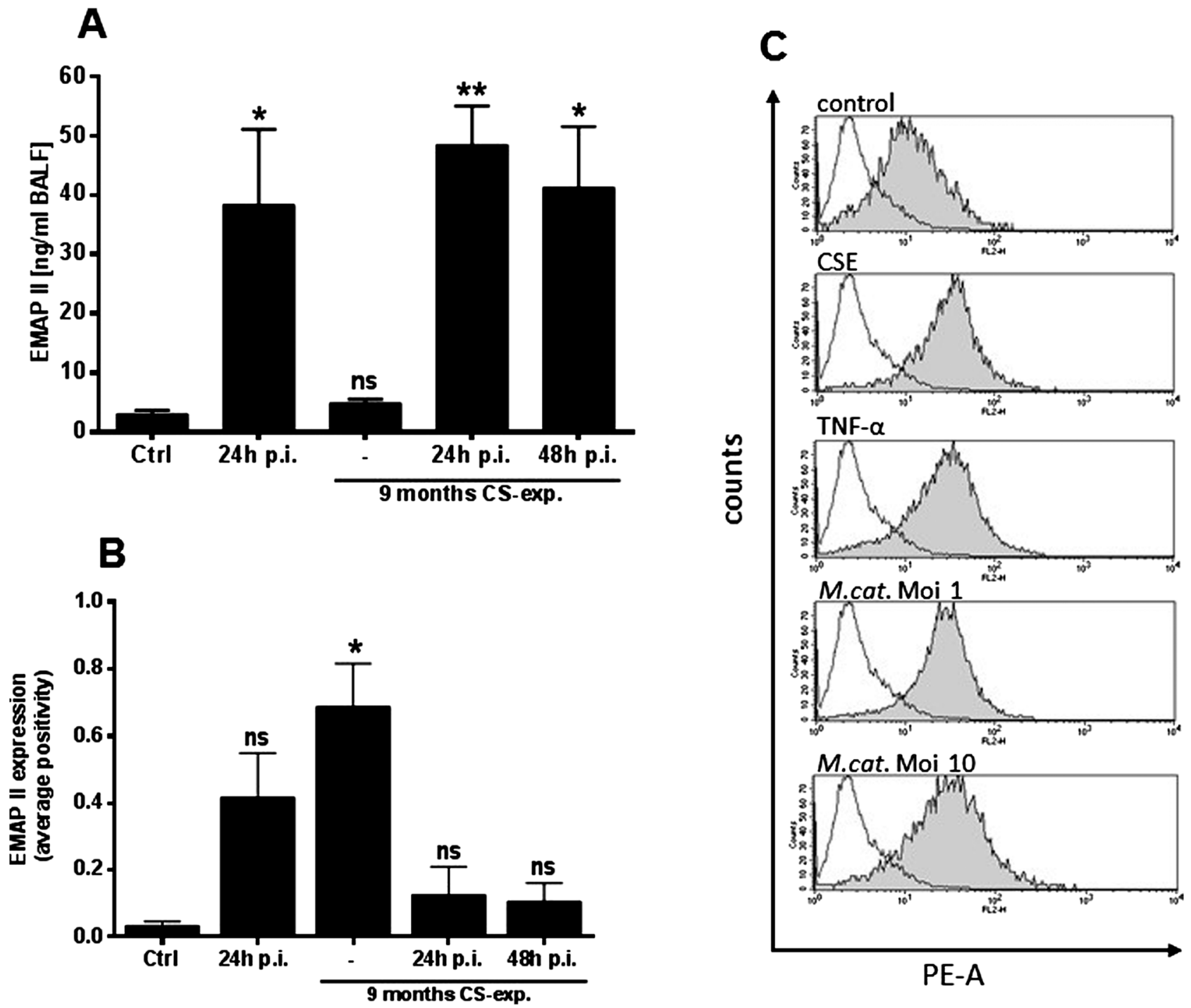

Figure 3. M. catarrhalis increases EMAPII expression in the BALF and lung tissue of long-term smoke-exposed mice and in primary mouse alveolar epithelial cells. (A) EMAPII expression measured as $\mathrm{ng} / \mathrm{mL}$ in the BALF and (B) EMAPII expression shown as average positivity in the lung tissue of mice with 24-h post infection with $M$. catarrhalis, after 9 months of CS-exposure alone, and with 24-h and 48-h post infection with M. catarrhalis upon prior smoke exposure and in control animals, $(n=3-4)$. Kruskal-Wallis test with comparison (Dunn's) of all groups versus Ctrl was used, and bars represent mean + SEM $\left({ }^{*} p<0.05, * * p<0.01\right)$. (C) Flow cytometry of EMAPII in primary mouse alveolar epithelial cells (filled histogram) left untreated or stimulated with CSE, TNF- $\alpha$, or M. catarrhalis (MOI 1 and MOI 10) for 2 h. Rat-IgG antibody was used as an isotype control (black line). The experiment was repeated three times, and a representative image was shown

\section{Discussion}

The results of our study demonstrate that M. catarrhalis infection acts as an additional independent risk factor for the induction of-emphysema inducing mechanisms in the lung of long-term smoke-exposed animals.

We showed that long-term smoke exposure alone induced significant damage to the murine bronchial epithelium and contributed to the development of emphysema. In particular, a marked rarefication of cilia on the cell surface of airway epithelial cells in smoke-exposed mice could be demonstrated, suggesting an increased risk for bacterial exacerbations. Importantly, acute $M$. catarrhalis infection independently contributed to the induction of emphysema developing mechanisms by increasing MMP9, MMP12, and EMAPII in the BALF, independent of prior smoke exposure.

It has been shown that long-term cigarette smoke exposure causes cilia shedding and reduced motility in murine models [3]. Our study is complementing these data by adding results obtained by a new method based on the quantification of the extent of cilia coverage on the cell surface of bronchial epithelium. In further histological analyses, we found significant lung damage and emphysema development, in addition to elevated levels of caspase- 3 in the lung tissue of long-term smoke-exposed animals. These data are in accordance with Aoshiba et al. who instilled active caspase- 3 in murine lungs and observed alveolar epithelial apoptosis being directly involved in emphysematous changes [9]. Nevertheless, our murine model with longterm smoke exposure is more closely related to the actual complex mechanisms of COPD pathogenesis. In the lungs of COPD patients, increased levels of EMAPII expression were recently identified to be an additional important emphysema-inducing mechanisms such as the induction of MMPs [11]. Our findings that M. catarrhalis infection leads to increased EMAPII expression in murine alveolar epithelial cells, as well as in the BALF of the murine lungs, suggest that acute $M$. catarrhalis exacerbation significantly contribute to the development of emphysema by the induction of this EMAPII mechanism.

Notably, we also identified $M$. catarrhalis infection as an independent risk factor for the increasing emphysema-inducing mechanisms by demonstrating a significant elevation of MMP9 and MMP12 expression in the BALF of M. catarrhalisinfected animals. 

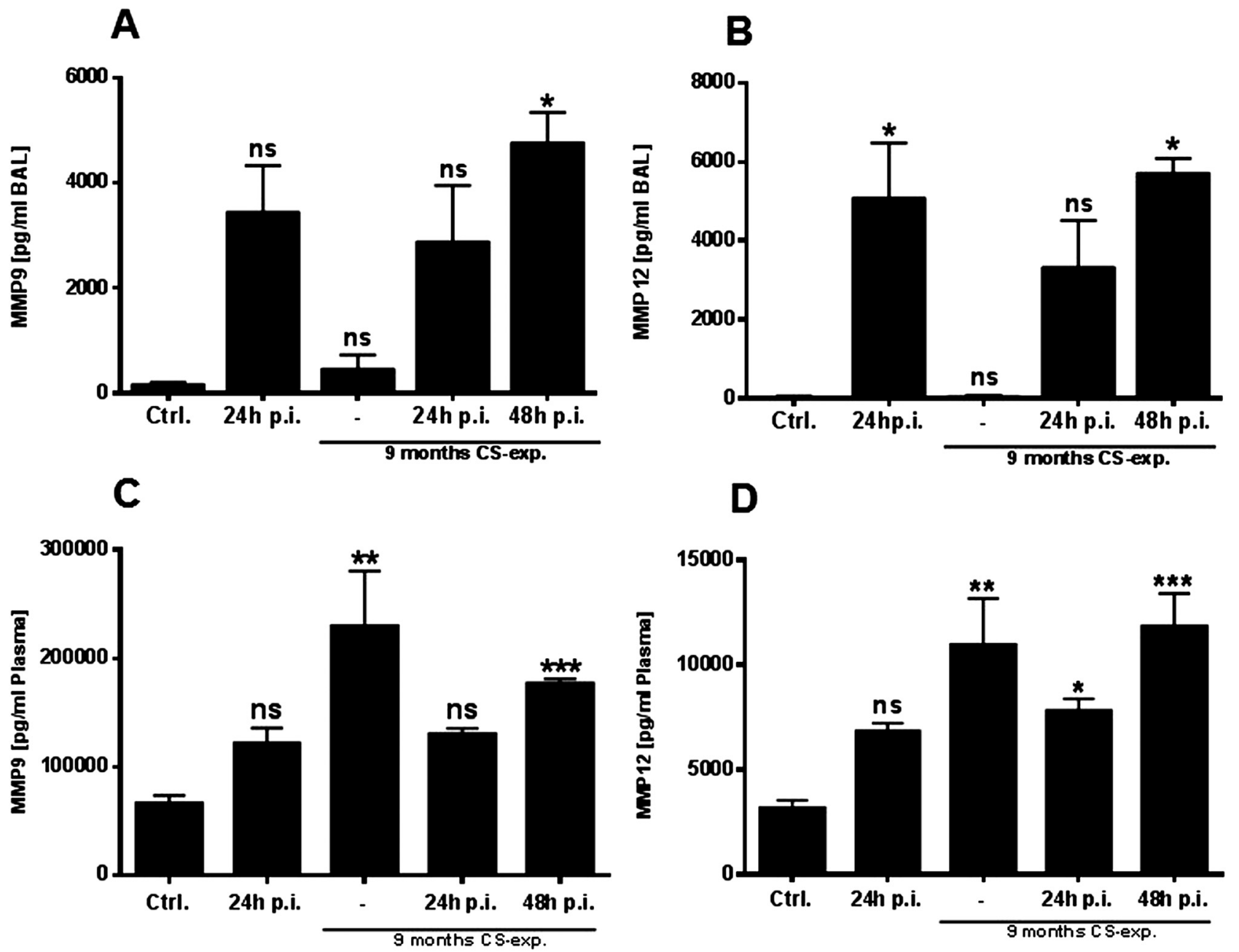

Figure 4. Acute $M$. catarrhalis airway infection increases MMP9 and MMP12 expression in the BALF and plasma of long-term smoke-exposed mice. (A) MMP9 and (B) MMP12 expression in the BALF and (C) MMP9 and (D) MMP12 expression in the plasma of mice with 24-h post infection with $M$. catarrhalis, after 9 months of CS-exposure alone, and with 24-h and 48-h post infection with M. catarrhalis upon prior smokeexposure and in control animals (BALF: $n=3-5$ and plasma: $n=6$ ). Kruskal-Wallis test with comparison (Dunn's) of all groups versus Ctrl. was used, and bars represent mean $+\operatorname{SEM}\left({ }^{*} p<0.05,{ }^{* *} p<0.01,{ }^{* * *} p<0.001\right)$

In COPD patients, which show bacterial colonization, MMPs were increased compared with patients without bacterial colonization [24]. Our data are also in accordance with Ohnishi et al., who found increased levels of MMP9 in human lungs with emphysema compared to healthy lungs [25]. The work from Churg et al. demonstrated that proteases, including MMP9, are damaging alveolar units by digestion of elastin and further structural proteins when being released from activated neutrophils and macrophages, and thus being the precursor of emphysema [26]. Furthermore, plasma levels of MMP 9 have been shown to be associated with disease severity in COPD and emphysema development [27]. While we found that acute $M$. catarrhalis infection led to an elevation of MMP9 and MMP12 in the BALF of smoke-exposed mice and elevated MMP9 expression in lung homogenate (Figure S3), we did not find evidence for an impact of solely long-term smoke exposure on the MMP expression in the BALF, suggesting $M$. catarrhalis as the main trigger for the intrapulmonary response. These data are in accordance with our finding of $M$. catarrhalis-induced EMAPII secretion into the airways, which we did not find in only smoke-exposed mice. In addition, we could demonstrate a great systemic effect of longterm smoke exposure alone or in combination with infection, when evaluating MMP9 and MMP12 expression in the plasma.

In summary, the results of our study suggest that, in addition to long-term CS exposure, acute exacerbations with
M. catarrhalis act as a considerable further risk factor for the development of emphysema by the additional induction of EMAPII solubilization and MMP9 and MMP12 secretions into the airways of smoke-exposed mice. In future clinical studies, these findings should be evaluated for their potential for preventing bacteria-induced exacerbations and reducing emphysema development.

\section{Supporting Information}

Detailed descriptions of the quantitative PCR analysis and caspase-3 staining and Figures $\mathrm{S} 1-\mathrm{S} 3$ are provided in the online supplement.

\section{Funding Sources}

This work was supported by the German Federal Ministry of Education and Research (German Asthma and COPD Network, COSYCONET: BMBF grant 01GI0888 and 03Z2JN22 to H.S.) and the Deutsche Forschungsgemeinschaft (SFB-TR 84, project C3 and C6 to M.W. and project Z1b to A.D.G).

\section{Authors' Contributions}

HS designed the study, and HS and BG supervised the conduct of the trial and data collection. KF, BG, JMD, CvL, CH, $\mathrm{AH}, \mathrm{OK}$, and $\mathrm{MHJ}$ carried out the data collection, and $\mathrm{KF}$ and 
HS analyzed the data. MC, RB, MV, ADG, and AH provided specific material and methods that were necessary to conduct the study. KF, BG, HS, and MW provided substantial contributions to the study conduct and design, and critically reviewed the manuscript. KF and HS drafted the manuscript, and all authors contributed substantially to its revision. All authors have provided their permission to publish the manuscript. This manuscript will be included in the doctoral thesis of Katja Fischer, Free University Berlin, Germany.

\section{Conflicts of Interest}

The authors report no conflicts of interest. The authors alone are responsible for the content and writing of the paper.

Acknowledgments. We greatly appreciate the technical assistance of Frauke Schreiber, Petra Schrade, and Kerstin Riskowsky and the support of Christina Dietert and Noelle Dahl regarding the histological evaluations and the EMAPII staining.

\section{References}

1. Barnes PJ. Chronic obstructive pulmonary disease: a growing but neglected global epidemic. PLoS Med. 2007;4:e112.

2. Baby MK, Muthu PK, Johnson P, Kannan S. Effect of cigarette smoking on nasal mucociliary clearance: A comparative analysis using saccharin test. Lung India. 2014;31:39-42.

3. Simet SM, Sisson JH, Pavlik JA, et al. Long-term cigarette smoke exposure in a mouse model of ciliated epithelial cell function. Am J Respir Cell Mol Biol. 2010;43:635-40.

4. Rangelov K, Sethi S. Role of infections. Clin Chest Med. 2014;35:87-100.

5. Friedman PJ. Imaging studies in emphysema. Proc Am Thorac Soc. 2008;5:494-500

6. Tuder RM, McGrath S, Neptune E. The pathobiological mechanisms of emphysema models: what do they have in common? Pulm Pharmacol Ther. 2003;16:67-78

7. Laurell C-B, Eriksson S. The Electrophoretic $\alpha$; 1-Globulin Pattern of Serum in $\alpha$; 1-Antitrypsin Deficiency. Scand J Clin Lab Invest. 1963;15:132-40.

8. Tuder RM, Petrache I. Review series Pathogenesis of chronic obstructive pulmonary disease. J Clin Invest. 2012;122:2749-55.

9. Aoshiba K, Yokohori N, Nagai A. Alveolar wall apoptosis causes lung destruction and emphysematous changes. Am J Respir Cell Mol Biol. 2003;28:555-62.

10. Clauss M, Voswinckel R, Rajashekhar G, et al. Lung endothelial monocyte-activating protein 2 is a mediator of cigarette smoke - induced emphysema in mice. J Clin Invest. 2011 Jun;121:2470-9. Epub 2011 May 16.
11. Hautamaki RD. Requirement for Macrophage Elastase for Cigarette Smoke-Induced Emphysema in Mice. Science. 1997;277:2002-04.

12. Chang Y, Al-Alwan L, Audusseau S, et al. Genetic deletion of IL-17A reduces cigarette smoke-induced inflammation and alveolar type II cell apoptosis. Am J Physiol Lung Cell Mol Physiol. 2014;306:L132-43. Epub 2013 Oct 4.

13. Vlahos R, Bozinovski S, Jones JE, et al. Differential protease, innate immunity, and NF-kappaB induction profiles during lung inflammation induced by subchronic cigarette smoke exposure in mice. Am J Physiol Lung Cell Mol Physiol. 2006;290:L931-45.

14. Alamoudi OS. Bacterial infection and risk factors in outpatients with acute exacerbation of chronic obstructive pulmonary disease: a 2-year prospective study. Respirology. 2007;12:283-7.

15. Gutbier B, Fischer K, Doehn J-M, et al. Moraxella catarrhalis induces an immune response in the murine lung that is independent of human CEACAM5 expression and long-term smoke exposure. Am J Physiol - Lung Cell Mol Physiol. 2015;309:L250-61.

16. Tanabe N, Muro S, Hirai $\mathrm{T}$, et al. Impact of exacerbations on emphysema progression in chronic obstructive pulmonary disease. Am J Respir Crit Care Med. 2011;183:1653-9.

17. Hendrix AY, Kheradmand F. The Role of Matrix Metalloproteinases in Development, Repair, and Destruction of the Lungs. Prog Mol Biol Transl Sci. 2017;148:1-29. Epub 2017 May 19. Review.

18. N'Guessan PD, Vigelahn M, Bachmann S, et al. The UspA1 protein of Moraxella catarrhalis induces CEACAM-1-dependent apoptosis in alveolar epithelial cells. J Infect Dis. 2007;195:1651-60.

19. Slevogt H, Seybold J, Tiwari KN, et al. Moraxella catarrhalis is internalized in respiratory epithelial cells by a trigger-like mechanism and initiates a TLR2- and partly NOD1-dependent inflammatory immune response. Cell Microbiol. 2007;9:694-707.

20. Zeldin DC, Wohlford-Lenane C, Chulada P, et al. Airway inflammation and responsiveness in prostaglandin $\mathrm{H}$ synthase-deficient mice exposed to bacterial lipopolysaccharide. Am J Respir Cell Mol Biol. 2001;25:457-65.

21. Klaile E, Klassert TE, Scheffrahn I, et al. Carcinoembryonic antigen (CEA)-related cell adhesion molecules are co-expressed in the human lung and their expression can be modulated in bronchial epithelial cells by non-typable Haemophilus influenzae, Moraxella catarrhalis, TLR3, and type I and II int. Respir Res. 2013;14:85.

22. Tuder RM, Petrache I, Elias J a, Voelkel NF, Henson PM. Apoptosis and emphysema: the missing link. Am J Respir Cell Mol Biol. 2003;28:551-4.

23. Barnett G, Jakobsen A, Tas M, Rice K, Carmichael J, Murray JC. Prostate Adenocarcinoma Cells Release the Novel Proinflammatory Polypeptide EMAP-II in Response to Stress Prostate Adenocarcinoma Cells Release the Novel Proinflammatory Polypeptide EMAP-II in Response to Stress 1. Cancer Res. 2000:2850-57.

24. Sethi S, Maloney J, Grove L, Wrona C, Berenson CS. Airway inflammation and bronchial bacterial colonization in chronic obstructive pulmonary disease. Am J Respir Crit Care Med. 2006;173:991-8.

25. Ohnishi K, Takagi M, Kurokawa Y, Satomi S, Konttinen YT. Matrix metalloproteinase-mediated extracellular matrix protein degradation in human pulmonary emphysema. Lab Invest. 1998;78:1077-87.

26. Churg A, Zay K, Shay S, et al. Acute cigarette smoke-induced connective tissue breakdown requires both neutrophils and macrophage metalloelastase in mice. Am J Respir Cell Mol Biol. 2002;27:368-74.

27. Koo HK, Hong Y, Lim MN, Yim JJ, Kim WJ. Relationship between plasma matrix metalloproteinase levels, pulmonary function, bronchodilator response, and emphysema severity. Int J COPD. 2016;11:1129-37. 